\title{
Persistent Associative Plasticity at an Identified Synapse Underlying Classical Conditioning Becomes Labile with Short-Term Homosynaptic Activation
}

\author{
긱iangyuan Hu and ${ }^{\circledR S}$ Samuel Schacher \\ Department of Neuroscience, Columbia University Medical Center, New York State Psychiatric Institute, New York, New York 10032
}

\begin{abstract}
Synapses express different forms of plasticity that contribute to different forms of memory, and both memory and plasticity can become labile after reactivation. We previously reported that a persistent form of nonassociative long-term facilitation (PNA-LTF) of the sensorimotor synapses in Aplysia californica, a cellular analog of long-term sensitization, became labile with short-term heterosynaptic reactivation and reversed when the reactivation was followed by incubation with the protein synthesis inhibitor rapamycin. Here we examined the reciprocal impact of different forms of short-term plasticity (reactivations) on a persistent form of associative long-term facilitation (PA-LTF), a cellular analog of classical conditioning, which was expressed at Aplysia sensorimotor synapses when a tetanic stimulation of the sensory neurons was paired with a brief application of serotonin on 2 consecutive days. The expression of short-term homosynaptic plasticity [post-tetanic potentiation or homosynaptic depression (HSD)], or short-term heterosynaptic plasticity [serotonin-induced facilitation or neuropeptide Phe-Met-Arg-Phe- $\mathrm{NH}_{2}$ (FMRFa)-induced depression], at synapses expressing PA-LTF did not affect the maintenance of PA-LTF. The kinetics of HSD was attenuated at synapses expressing PA-LTF, which required activation of protein kinase C (PKC). Both PA-LTF and the attenuated kinetics of HSD were reversed by either a transient blockade of PKC activity or a homosynaptic, but not heterosynaptic, reactivation when paired with rapamycin. These results indicate that two different forms of persistent synaptic plasticity, PA-LTF and PNA-LTF, expressed at the same synapse become labile when reactivated by different stimuli.
\end{abstract}

Key words: Aplysia; heterosynaptic activity; homosynaptic activity; protein kinase C; protein synthesis; reconsolidation

Significance Statement

Activity-dependent changes in neural circuits mediate long-term memories. Some forms of long-term memories become labile and can be reversed with specific types of reactivations, but the mechanism is complex. At the cellular level, reactivations that induce a reversal of memory must evoke changes in neural circuits underlying the memory. What types of reactivations induce a labile state at neural connections that lead to reversal of different types of memory? We find that a critical neural connection in Aplysia, which is modified with different stimuli that mediate different types of memory, becomes labile with different types of reactivations. These results provide insights for developing strategies in alleviating maladaptive memories accompanying anxiety disorders.

\section{Introduction}

Long-term memory can be reversed by timely interventions during the induction, consolidation, or maintenance phase (Squire

\footnotetext{
Received May 27, 2015; revised 0ct. 6, 2015; accepted Nov. 5, 2015.

Author contributions: J.H. and S.S. designed research; J.H. performed research; J.H. and S.S. analyzed data; J.H. and S.S. wrote the paper.

This work was supported by National Institutes of Health (NIH) Grant MH060387. Animals were provided by the National Center for Research for Aplysia at the University of Florida in Miami, which is supported by NIH Grant RR-10294. We thank Dr. Amir Levine for comments, Dr. Tim Requarth for proofreading this manuscript, and Kerry Adler for technical assistance.

The authors declare no competing financial interests.

Correspondence should be addressed to Jiangyuan Hu, Department of Neuroscience, Columbia University Medical Center, New York State Psychiatric Institute, 1051 Riverside Drive, New York, NY 10032. E-mail: jh2004@cumc.columbia.edu.
}

and Alvarez, 1995; McGaugh, 2000; Kandel, 2001; Johansen et al., 2011). Persistent memories and certain forms of synaptic plasticity become labile with specific reactivations and express reconsolidation blockade, a reversal when a specific reactivation is paired with manipulations that inhibit protein synthesis or degradation, gene expression, specific kinases such as various isoforms of protein kinase $\mathrm{C}$ (PKC), or receptor activation (Nader et al., 2000; Kelly et al., 2003; Morris et al., 2006; Lubin and Sweatt, 2007; Shema et al., 2007; Dong et al., 2008; Zhang et al., 2010; Cai et al., 2011, 2012; Kaang and Choi, 2011; Sacktor, 2011; Lee et al., 2012; Da Silva et al., 2013; Li et al., 2013; Alberini and Kandel, 
2014; Chen et al., 2014; Hu and Schacher, 2014; Fiumara et al., 2015). Because different forms of plasticity at specific synapses induced by different patterns of stimuli contribute to a given memory, it is generally difficult to determine what forms of reactivation at which specific synapses induce a labile state that makes a memory or its underlying plasticity vulnerable to reversal.

Withdrawal reflexes in Aplysia, which undergo long-term nonassociative (sensitization) and associative (classical conditioning) forms of learning and memory (Pinsker et al., 1973; Carew et al., 1981, 1983; Frost et al., 1985; Buonomano and Byrne, 1990), can express reconsolidation blockade (Chen et al., 2014). Repeated sensitizing stimuli, which activate modulatory neurons releasing serotonin [5-hydroxytryptamine (5-HT)], or repeated direct applications of 5-HT evoke a nonassociative form of long-term facilitation (NA-LTF) at sensorimotor synapses (Brunelli et al., 1976; Frost et al., 1985; Montarolo et al., 1986; Glanzman et al., 1989; Mackey et al., 1989; Marinesco et al., 2006). Persistent nonassociative LTF (PNA-LTF) of sensorimotor synapses or sensitization of the reflex lasting more than 1 week is produced with multiple days of 5-HT applications or of sensitizing stimuli (Pinsker et al., 1973; Kandel, 2001; Hu et al., 2011). PNA-LTF at Aplysia sensorimotor synapses is coexpressed with various forms of short-term plasticity and undergoes reconsolidation blockade when heterosynaptic [5-HT or PheMet-Arg-Phe- $\mathrm{NH}_{2}$ (FMRFa)], but not homosynaptic [posttetanic potentiation (PTP) or homosynaptic depression (HSD)], reactivation is followed by an incubation with the protein synthesis inhibitor rapamycin (Hu and Schacher, 2014). Protein kinase $\mathrm{C}$ (PKC) activity also is required for consolidation of long-term sensitization and non-associative LTF (Villareal et al., 2009; Hu et al., 2011; Chen et al., 2014; Fiumara et al., 2015). What types of reactivations or inhibitors of signaling cascades would make associative forms of memory or its underlying plasticity labile and susceptible to reversals?

Activity or tactile input to the sensory neurons plus the actions of 5-HT affect the strength of sensorimotor synapses and contribute to classical conditioning (Hawkins et al., 1983; Kandel et al., 1983; Walters and Byrne, 1983; Eliot et al., 1994). In cell culture, one temporal pairing of a brief tetanic stimulation in the sensory neuron with an application of 5-HT evokes an associative form of LTF (A-LTF), a cellular analog of classical conditioning of the reflexes, that lasts $>24 \mathrm{~h}$ and requires new macromolecular synthesis (Schacher et al., 1997; Hu et al., 2007).

Here we address the following questions: Does the sensorimotor synapse express a persistent form of associative LTF (PALTF)? Does constitutive PKC activity contribute to PA-LTF? What type of reactivation makes PA-LTF labile and susceptible to reversal? We found that PA-LTF at sensorimotor synapses was produced by pairing activity in the sensory neurons with application of 5-HT on 2 consecutive days and was coexpressed with different forms of short-term plasticity. The kinetics of HSD was attenuated at synapses expressing PA-LTF that required PKC activity. Moreover, a transient blockade of PKC activity reversed PA-LTF. The synapses expressing PA-LTF became labile and susceptible to reversals with homosynaptic, but not heterosynaptic, reactivation. Thus, different forms of persistent plasticity expressed at the same synapse become labile when reactivated by different stimuli.

\section{Materials and Methods}

Cell culture and electrophysiology. Sensory neurons were isolated from pleural ganglia dissected from adult animals (60-80 g; Aplysia californica, which are hermaphrodite with both male and female sexual organs), and the L7 motor neurons were isolated from juvenile (2 $\mathrm{g}$ ) abdominal ganglia and maintained in coculture for 7-11 d (Hu et al., 2011). Each coculture contained one sensory neuron and one L7. Standard electrophysiological techniques were used to record the EPSP amplitudes evoked in L7. L7s were held at $-80 \mathrm{mV}$, and the EPSP amplitudes were recorded before and after various treatments at the time points indicated in the figures highlighting the experimental protocols. Each sensory neuron was stimulated with a brief depolarizing pulse to evoke an action potential using an extracellular electrode placed near the cell body of the sensory neuron. To clearly measure the change of the EPSP amplitudes at subsequent time points, especially after treatments that produced significant increases in the synaptic strength that could result in action potential responses, cultures with initial EPSP amplitudes of $15-30 \mathrm{mV}$ were selected for all experiments. The few cultures $(<5 \%)$ with synapses that responded with an action potential as the EPSP after treatment were not used for analyses. The culture medium was changed every other day with medium containing 50\% filtered hemolymph and 50\% Leibowitz-15 (L15; Sigma). All cultures were maintained at $18^{\circ} \mathrm{C}$.

PA-LTF. A-LTF lasting $>24 \mathrm{~h}$ was evoked by a single pairing of stimuli, as reported previously (Schacher et al., 1997; Hu et al., 2007). In brief, after recording the synaptic baseline of all synapses on day 4 , some sensory neurons were stimulated on day 5 with a tetanus $(20 \mathrm{~Hz}$ for $2 \mathrm{~s})$ that was followed immediately by an application of 5-HT ( $5 \mu \mathrm{M}$; Sigma) for 5 min (Fig. 1). Cultures were rinsed with L15-sea water and then with culture medium (50\% filtered hemolymph and 50\% L15). PA-LTF, lasting $>6 \mathrm{~d}$, was produced by two pairings of a presynaptic tetanus with an application of 5-HT on 2 consecutive days (days 4 and 5; Fig. 1). Control cultures received mock treatments or unpaired stimulation where the tetanus and application of 5-HT were separated by 5 min (unpaired). The strengths of the synapses were re-examined on day 7 and at subsequent time points, as indicated in the protocol portion of the figures.

Short-term plasticity and interaction with PA-LTF. After recording the EPSP amplitude on day 7, some cultures (control or paired stimuli groups) were exposed to either $5 \mu \mathrm{M} 5-\mathrm{HT}$ or $1 \mu \mathrm{M}$ FMRFa (Sigma) for 5 min to evoke short-term heterosynaptic plasticity (Hu and Schacher, 2014). The EPSP amplitudes were re-examined on subsequent days. In other cultures, the EPSPs were recorded $1 \mathrm{~min}$ after washout of the neuromodulator to monitor the magnitude of short-term plasticity. Other cultures received an application of the protein synthesis inhibitor rapamycin (100 nm; Sigma) for $2 \mathrm{~h}$ beginning immediately after the washout of the neuromodulator. As above, the EPSP amplitudes were re-examined on subsequent days. In addition, HSD was monitored on day 8 and sometimes on day 10 (data not shown).

The same general procedure was followed to induce short-term homosynaptic plasticity and to examine the interaction between short-term homosynaptic activity and PA-LTF. After recording the EPSP amplitudes on day 7, sensory neurons (control or paired stimuli groups) were stimulated to fire a single action potential at $20 \mathrm{~s}$ intervals for 10 stimuli to produce HSD. In other cultures, the sensory neurons (control or paired stimuli groups) were stimulated at $20 \mathrm{~Hz}$ for $2 \mathrm{~s}$ to produce PTP. Other cultures were exposed to $100 \mathrm{~nm}$ rapamycin for $2 \mathrm{~h}$ beginning immediately after HSD, PTP, or controls. The amplitudes of the EPSPs were monitored on subsequent days. In addition, HSD was monitored on day 8 and sometimes on day10 (data not shown). To monitor the extent of PTP, the EPSP amplitudes were monitored $1 \mathrm{~min}$ after the tetanus.

To examine any change in the kinetics of HSD on day 7, some cultures (control or paired stimuli groups) were exposed to the PKC inhibitor (PKCI) chelerythrine $[10 \mu \mathrm{M}$; dissolved in $0.1 \%$ dimethyl sulfoxide (DMSO); Sigma] or the protein kinase A inhibitor (PKAI) KT5720 (10 $\mu \mathrm{M}$; Sigma) for $30 \mathrm{~min}$ after recording the initial EPSP amplitude before low-frequency stimulation that results in HSD. To test the role of constitutive kinase activity in PA-LTF, chelerythrine or KT5720 was added to other cultures for $2 \mathrm{~h}$ on day 7 after testing the EPSP amplitudes, and the EPSP amplitudes were re-examined on days 8 and 10. The kinetics of HSD was examined on day 8.

Quantification and data analysis. All data are expressed as the mean \pm SEM produced by the indicated treatments. The EPSP amplitude was measured in millivolts $(\mathrm{mV})$, and cultures were assigned to different experimental groups to ensure that the average of the initial EPSP ampli- 

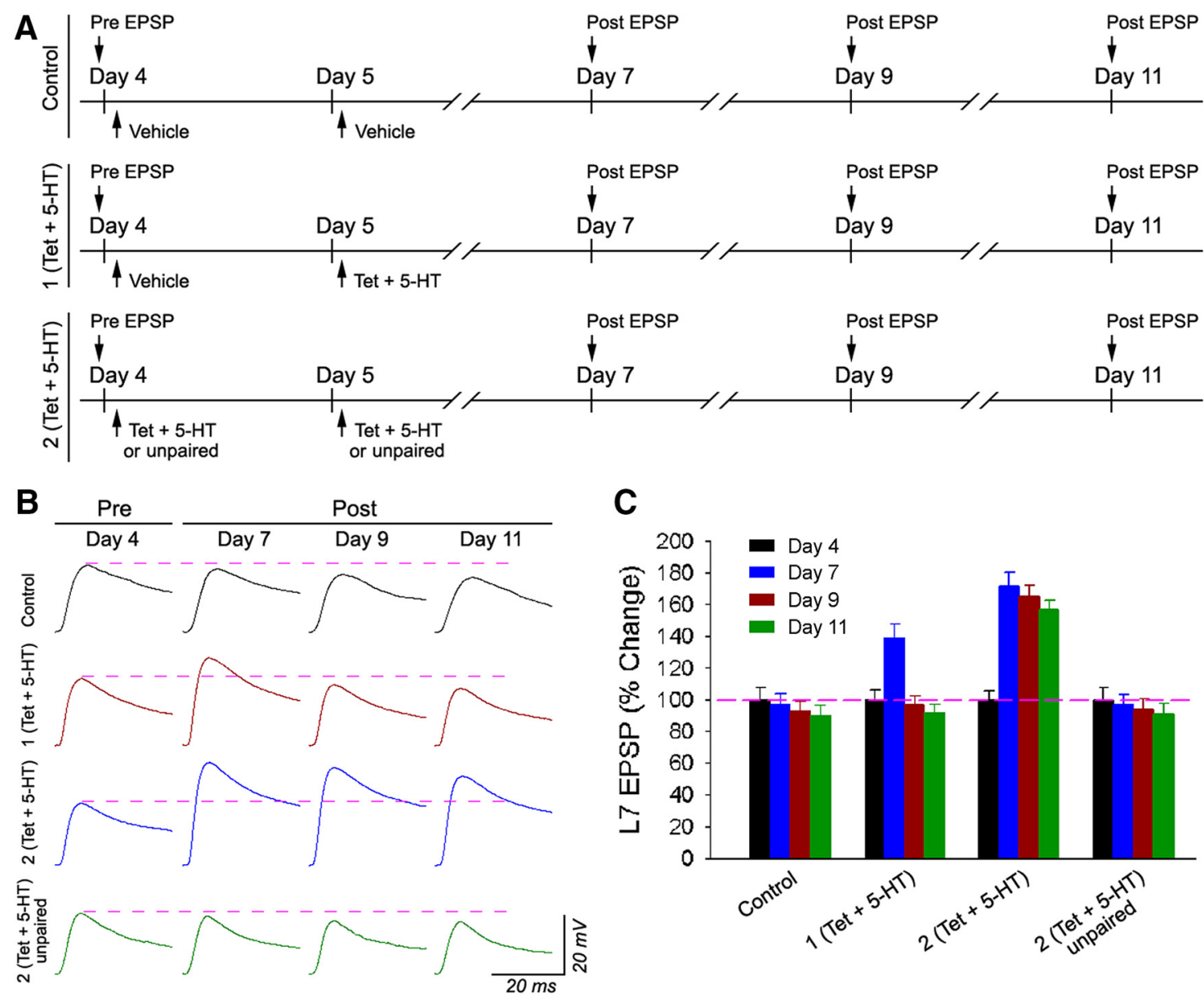

Figure 1. PA-LTF is evoked with two pairings of tetanic stimulation in the sensory neurons and application of 5-HT. $A$, Experimental protocol. Cultured cells were divided into four groups after an initial recording of the EPSPs on day 4. The control group (Control) received mock applications of seawater on days 4 and 5 . A second group [1 (Tet +5 -HT)] received a mock application on day 4 and a single pairing of tetanus ( $20 \mathrm{~Hz}$ for $2 \mathrm{~s}$ stimulated in the sensory neurons) followed immediately by a bath application of 5 -HT $(5 \mu \mathrm{m})$ for 5 min on day 5 . A third group [2 (Tet +5 -HT)] received two pairings of presynaptic tetanus with 5-HT application, the first on day 4 and the second on day 5 . The fourth group [2 (Tet +5 -HT) unpaired] received the same stimuli as the third group, but the tetanic stimulation preceded each application of 5 -HT by $5 \mathrm{~min}$. The EPSPs were re-examined on days 7, 9, and 11. B, Sample EPSP traces for each treatment. The dashed lines represent the synaptic baseline. A single pairing evoked a transient LTF on day 7, whereas two pairings evoked a persistent facilitation to day 11. Control and unpaired groups showed little change in the EPSP amplitudes over $7 \mathrm{~d}$. C, Summary of the changes in the EPSPs evoked by control and experimental groups. A two-factor ANOVA (treatment and time) indicated a significant difference between the groups $\left(F_{(9,99)}=85.157 ; p<0.001\right)$. Individual comparisons indicated that both 1 (Tet +5 -HT) and 2 (Tet +5 -HT) produced significant increases in the EPSP amplitudes on day 7 over the control $(F=3.196$ and $13.35 ; p<0.05$ and 0.01 ) and 2 (Tet +5 -HT) unpaired $(F=2.976$ and $11.028 ; p<0.05$ and 0.01 ) groups. Only 2 (Tet +5 -HT) evoked significant increases on days 9 and 11 compared with the control $(F=17.521$ and 19.665; $p<0.05$ and 0.01$), 1$ (Tet $+5-H T)(F=15.462$ and 18.723; $p<0.01$ and 0.01$)$, or 2 (Tet +5 -HT) unpaired $(F=13.817$ and $15.794 ; p<0.01$ and 0.01 ) groups. Control, 1 (Tet $+5-\mathrm{HT}$ ), and 2 (Tet +5 -HT) unpaired treatments were not significantly different from each other on day 9 ( Fvalues between 0.005 and $0.049 ;$ all $p$ values $>0.8$ ) or day 11 ( $F$ values between 0.002 and 0.009 ; all $p$ values $>0.9$ ).

tudes for all groups on day 4 was comparable. To measure the amplitude of PA-LTF, the EPSP amplitudes on day 4 were normalized as $100 \%$. The changes in the EPSP amplitudes were measured by dividing the EPSP amplitudes on day 7 and later time points by the EPSP amplitude on day 4 multiplied by $100 \%$. No change in the EPSP amplitude was scored as $100 \%$. To measure short-term heterosynaptic plasticity produced by the brief exposure to 5-HT or FMRFa, and homosynaptic plasticity evoked by PTP or HSD, the initial EPSP amplitude on day 7 or day 8 was normalized as $100 \%$. The change in the EPSP amplitudes associated with short-term plasticity was expressed by dividing the amplitude of each EPSP by the amplitude of the first EPSP measured on day 7 or day 8 multiplied by $100 \%$. A two-factor ANOVA (treatment $\times$ repeated measure or time) or a one-factor ANOVA (treatment) was used to assess overall significant differences, and the Scheffé $F$ test was used to gauge significant differences between individual treatments.

\section{Results}

\section{Sensorimotor synapses express PA-LTF}

Long-term nonassociative facilitation of sensorimotor synapses requires both multiple exposures to a "sensitizing" stimulus, such as exposure to the neuromodulatory transmitter 5-HT, and new macromolecular synthesis, new gene and protein expression, to initiate and maintain synaptic changes (Montarolo et al., 1986; Schacher et al., 1988; Bailey et al., 1992; Kandel, 2001; Byrne and Hawkins, 2015). A transient form of NA-LTF, produced by five brief exposures to 5 -HT over a $90 \mathrm{~min}$ period, requires new macromolecular synthesis but reverses after $3 \mathrm{~d}$ (Hu et al., 2011; Hu and Schacher, 2014). A more persistent form of NA-LTF (PNALTF) of sensorimotor synapses (lasting $>6 \mathrm{~d}$ ) is evoked by 2 consecutive days of 5-HT applications ( 5 min incubation of $5 \mu \mathrm{M}$ 5-HT applied five times at 20 min intervals on each day; $\mathrm{Hu}$ et al., 2011). A-LTF, a cellular analog of classical conditioning, is produced by a single pairing of action potential activity in a sensory neuron (the conditioned stimulus consisting of a brief tetanus of action potentials fired in the presynaptic sensory neuron) with a sensitizing or noxious stimulus (the unconditioned stimulus, consisting of a brief application of 5-HT). A-LTF requires macromolecular synthesis and lasts $>24 \mathrm{~h}$ (Sun and Schacher, 1996a; 
A $5-H T$
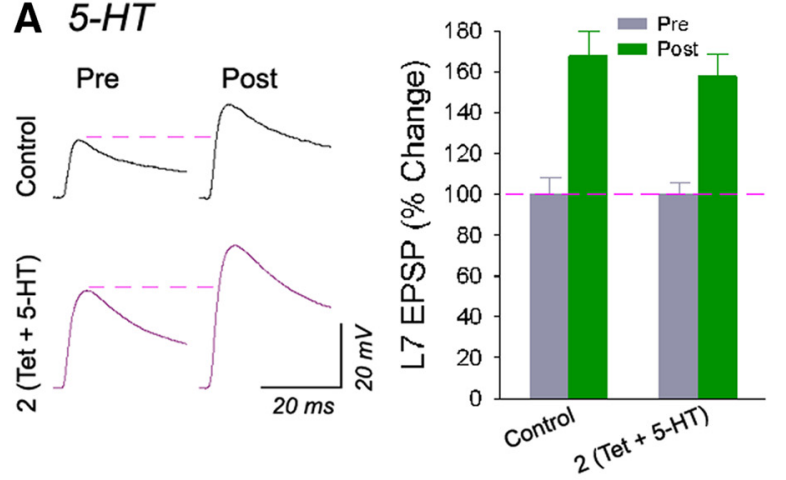

C PTP<smiles>CC1CCC(CO)C1[O+]</smiles>
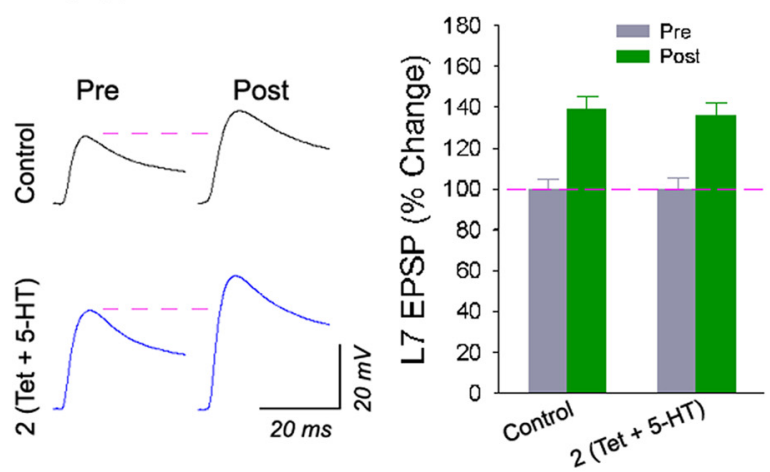
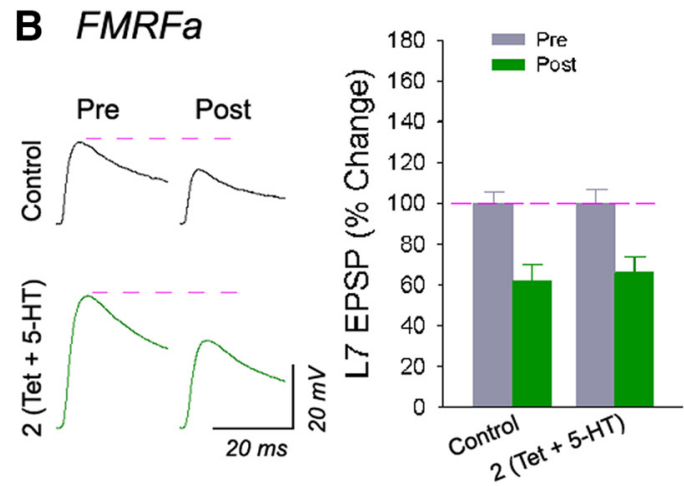

D $H S D$

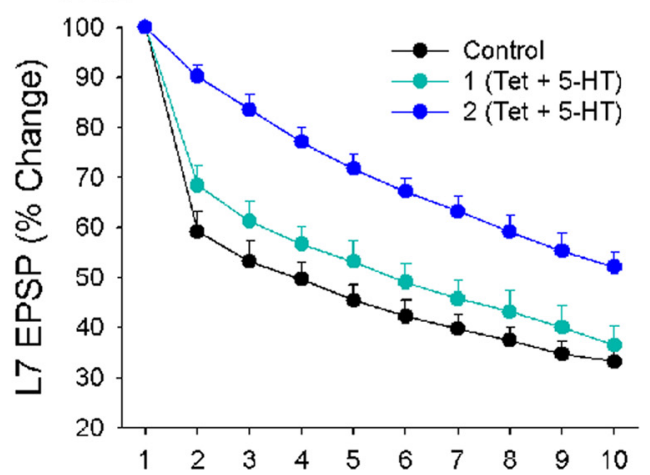

Figure 2. Various forms of short-term plasticity are coexpressed with PA-LTF. A-C, Expression of PA-LTF did not affect the expression of short-term heterosynaptic facilitation induced by 5-HT $(\boldsymbol{A})$, heterosynaptic depression by FMRFa $(\boldsymbol{B})$, or homosynaptic facilitation evoked by PTP $(\boldsymbol{C})$ on day 7 . On the left are sample traces of EPSPs evoked in L7 at control synapses or synapses expressing PA-LTF before (Pre) and $1 \mathrm{~min}$ after (Post) a 5 min application of 5 -HT $(\boldsymbol{A})$, a $5 \mathrm{~min}$ application of FMRFa $(\boldsymbol{B})$, or a tetanus ( $20 \mathrm{~Hz}$ for $2 \mathrm{~s}$ ) in the sensory neurons ( $\boldsymbol{C}$. Dashed lines represent the baseline (Pre amplitude on day 7). The histograms in $A-$ Csummarize the short-term changes in the EPSP amplitudes. Two-factor ANOVAs indicated that there were no significant differences in the short-term changes evoked at synapses expressing PA-LTF compared with changes at control synapses after 5 -HT $\left(F_{(1,10)}=2.667 ; p>0.1\right)$, FMRFa $\left(F_{(1,11)}=0.702 ; p>0.4\right), 0$ PTP $\left(F_{(1,14)}=0.047 ; p>0.8\right)$. However, one-factor ANOVAs indicated significant short-term changes produced by 5-HT, FMRFa, and PTP at synapses expressing PA-LTF ( $F$ values between 34.623 and 127.661 ; all $p$ values $<0.01$ ) or at control synapses ( $F$ values between 41.336 and 119.618; all $p$ values $<0.01$ ). D, Expression of PA-LTF attenuates HSD evoked by low-frequency stimulation of sensory neurons (10 stimuli) on day 7. The first EPSP amplitude for all treatments was normalized as $100 \%$. A two-factor ANOVA indicated a significant effect on the kinetics of HSD $(F(16,152)=6.077 ; p<0.001)$. Individual comparisons showed a significant effect evoked by 2 (Tet $+5-\mathrm{HT})$ compared with control $(F=9.873 ; p<0.001)$ and 1 (Tet +5 -HT) $(F=4.779 ; p<0.001)$. Control and 1 (Tet +5 -HT) were not significantly different $(F=1.674 ; p>0.2)$.

Hu et al., 2007). How long does A-LTF last, and can we produce more persistent changes with additional paired stimulation? We first compared the duration of synaptic facilitation evoked by one pairing of a presynaptic tetanus $(20 \mathrm{~Hz}$ for $2 \mathrm{~s})$ immediately followed by a brief application of 5-HT ( $5 \mathrm{~min}, 5 \mu \mathrm{M})$ versus two pairings of a presynaptic tetanus plus 5 -HT application on 2 consecutive days or 2 unpaired stimuli ( $5 \mathrm{~min}$ separation between the presynaptic tetanus and the 5-HT application) on 2 consecutive days (Fig. 1).

Two pairings of presynaptic tetanus plus 5-HT produced a persistent facilitation lasting $>6 \mathrm{~d}$, whereas a single pairing evoked a facilitation lasting only $3 \mathrm{~d}$ (Fig. 1). Unpaired stimuli on 2 consecutive days produced little or no facilitation at any time point (Fig. 1). On day 7, the EPSP amplitudes increased significantly after one pairing $(n=8 ; 139 \pm 8.9 \%)$ and after two pairings $(n=15 ; 172 \pm 8.5 \%)$ compared with controls $(n=8 ; 97 \pm$ $7.1 \%$; $p<0.05$ and $p<0.01$, respectively) and after two unpaired stimuli ( $n=6$; $97 \pm 6.6 \% ; p<0.05$ and $p<0.01$, respectively). By days 9 and 11 ( $6 \mathrm{~d}$ after stimulation), only two pairings evoked a significant increase $(p<0.01$ in all cases $)$ in the EPSP amplitude $(165 \pm 7.5 \%$ and $157 \pm 6.2 \%)$ compared with controls $(93 \pm$ $6.3 \%$ and $90 \pm 6.3 \%)$, one pairing ( $97 \pm 5.6 \%$ and $92 \pm 5.3 \%$ ), or two unpaired stimuli ( $94 \pm 6.7 \%$ and $91 \pm 6.7 \%$ ). Thus, associative LTF has a transient form (A-LTF) evoked by a single pairing that reverses spontaneously after $3 \mathrm{~d}$ and a persistent form (PA-
LTF) lasting $>6 \mathrm{~d}$ that is produced by two pairings of presynaptic tetanus plus 5 -HT. The time courses of the associative forms of facilitation, A-LTF and PA-LTF, parallel the time courses of the nonassociative forms of LTF (Hu and Schacher, 2014) evoked by repeated applications of 5-HT over $1 \mathrm{~d}$ (NA-LTF) and over $2 \mathrm{~d}$ (PNA-LTF).

\section{PA-LTF is co-expressed with short-term plasticity}

We next examined whether bidirectional short-term homosynaptic and heterosynaptic plasticity were coexpressed at synapses expressing PA-LTF (Fig. 2). The different forms of short-term plasticity at sensorimotor synapses are the cellular analogs of short-term forms of learning and memory, including habituation and sensitization (Byrne and Hawkins, 2015). After recording the EPSP amplitudes on day 7, control synapses or synapses expressing PA-LTF were reactivated by short-term heterosynaptic activity (a brief application of 5-HT or FMRFa) or homosynaptic activity (PTP or HSD; Fig. 2). The synaptic strengths were monitored 1 min after the application of either 5-HT or FMRFa or after tetanus.

The expression of short-term heterosynaptic plasticity was not affected at sensorimotor synapses expressing PA-LTF $48 \mathrm{~h}$ after the second pairing of presynaptic tetanus plus 5-HT (Fig. $2 A, B)$. The application of 5-HT produced comparable increases in the EPSP amplitudes $(p>0.1)$ in both the control synapses 
A
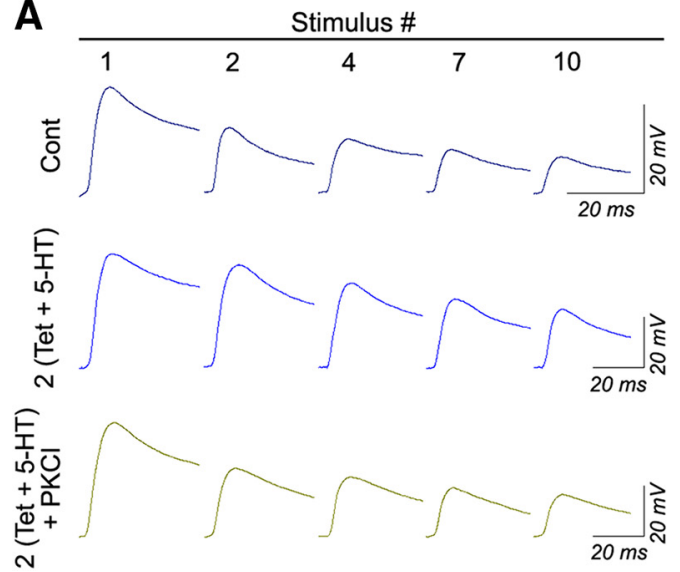

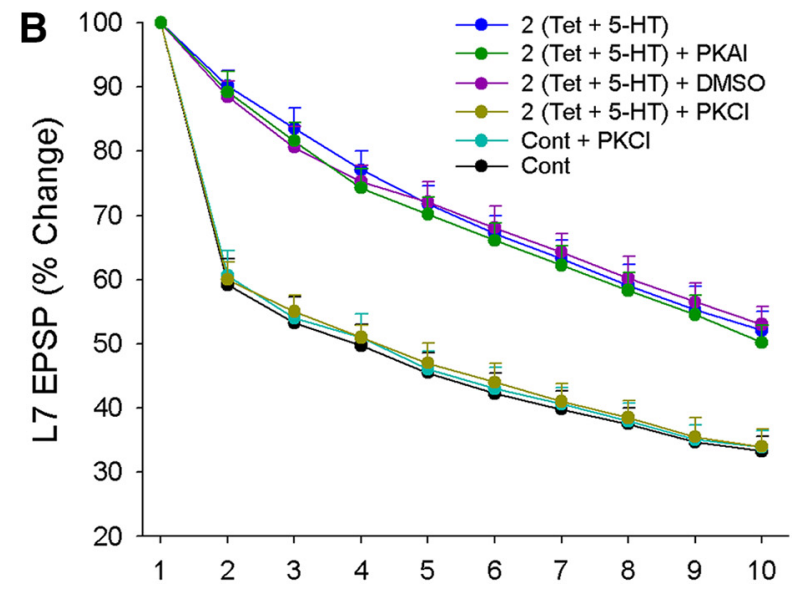

Figure 3. PKC activity is required for the attenuated kinetics of HSD that accompany PA-LTF. Cultures were exposed to PKC inhibitor chelerythrine (PKCl; $10 \mu \mathrm{m}$ ) or PKA inhibitor KT5720 (PKAl; $10 \mu \mathrm{m}$ ) or received vehicle treatment for $30 \mathrm{~min}$ after recording the initial EPSP amplitude and before HSD stimuli on day 7. A, Sample traces of EPSPs at different stimulus numbers are compared for cultures treated with control (Cont), 2 (Tet $+5-\mathrm{HT}$ ), and 2 (Tet $+5-\mathrm{HT})+$ PKCl. The significant decline in EPSPs was absent at synapses treated with 2 (Tet +5 -HT). The decline observed with controls was comparable to that of synapses expressing PA-LTF plus incubation with chelelythrine. $\boldsymbol{B}$, Summary of the changes in the kinetics of HSD. The kinetics of HSD were attenuated for cultures treated with 2 (Tet $+5-\mathrm{HT}), 2$ (Tet +5 - HT) + DMSO, and 2 (Tet $+5-\mathrm{HT})+$ PKAl, whereas treatment with PKCl $[2$ (Tet +5 -HT) + PKCl] reversed the attenuated kinetics produced by 2 (Tet + $5-\mathrm{HT})$ so that it was not different from those observed in the control and Cont $+\mathrm{PKCl}$ groups. A two-factor ANOVA indicated a significant effect on the kinetics of HSD $\left(F_{(40,280)}=4.047 ; p<0.001\right)$. Individual comparisons showed that the attenuated kinetics of HSD in 2 (Tet +5 -HT), 2 (Tet +5 -HT) + DMSO, and 2 (Tet +5 -HT) + PKAI were not significantly different from each other (Fvalues between 0.419 and 1.447 ; all $p$ values $>0.15$ ) but were significantly different from those in 2 (Tet +5 - HT) $+\mathrm{PKCl}(F=6.506, p<0.001 ; F=2.474, p<0.02 ; F=7.182, p<0.001)$, control $(F=9.873, p<0.001 ; F=4.072, p<0.001 ; F=15.436, p<0.001)$, or Cont $+\operatorname{PKCl}(F=7.262, p<0.001 ; F=2.886, p<0.008 ; F=11.078, p<0.001)$. Treatments that did not attenuate HSD kinetics were not significantly different from each other ( $F$ values between 0.063 and 0.101 ; all $p$ values $>0.9$ ).

$(n=6 ; 173 \pm 11.8 \%)$ and the synapses expressing PA-LTF $(n=$ $6 ; 158 \pm 10.6 \%)$. Similarly, the brief application of FMRFa resulted in a depression of the EPSP amplitudes to $66 \pm 7.8 \%$ at synapses expressing PA-LTF $(n=6)$ that was not significantly different from the depression in the EPSP amplitude to $62 \pm$ $8.0 \%$ in controls $(n=7 ; p>0.4$; Fig. $2 B)$. The overall depression evoked by FMRFa at synapses expressing PA-LTF resulted in the synaptic strength returning to the amplitude recorded on day 4 . Thus, the functional expression of short-term heterosynaptic plasticity was not altered by the expression of PA-LTF.

Short-term homosynaptic facilitation (PTP) was not affected by the expression of PA-LTF (Fig. 2C). Tetanic stimulation of the sensory neuron evoked an increase in the EPSP amplitude of $136 \pm 6.1 \%$ at sensorimotor synapses expressing PA-LTF $(n=8)$ that was not significantly different from the increase of $139 \pm$ $6.3 \%$ at control synapses $(n=8 ; p>0.8)$. Thus, the functional expression of PTP was unaffected by the expression of PA-LTF. The expression of various forms of short-term plasticity at synapses already expressing an associative form of long-term plasticity (with properties that appear comparable to the short-term plasticity observed at naive synapses) suggests that a new synaptic baseline is established $48 \mathrm{~h}$ after stimuli that produce a persistent form of plasticity.

Short-term HSD was detected at synapses expressing PALTF, but the kinetics of HSD was attenuated significantly (Fig. $2 D)$. The rate of decline of the EPSP amplitude was significantly reduced when the synapses express PA-LTF $(n=8)$ compared with controls $(n=7 ; p<0.001)$ or nonpersistent A-LTF evoked after one pairing $(n=7 ; p<0.001)$. By the last stimulus to produce HSD, the EPSP amplitudes declined to $33 \pm 2.3 \%$ for control, to $37 \pm 3.9 \%$ after one pairing, but only to $52 \pm 2.9 \%$ after two pairings. Unlike the situation after stimuli that evoke PNA-LTF (Hu and Schacher, 2014), the expression of PA-LTF alters the properties of sensorimotor synapses during low-frequency stimulation of the sensory neurons.
The attenuated kinetics of HSD at sensorimotor synapses expressing PA-LTF parallels the kinetics of HSD induced at the same synapses after an application of 5-HT, an incubation with phorbol ester, or firing two to four action potentials in the sensory neurons that increase PKC activity (Sun and Schacher, 1996b; Wan et al., 2012). We therefore tested whether the attenuated kinetics of HSD at synapses expressing PA-LTF were affected by the presence of a PKC or PKA inhibitor on day 7 (Fig. 3). Before the stimuli inducing HSD, some cultures were incubated for $30 \mathrm{~min}$ in $10 \mu \mathrm{M}$ chelerythrine (PKCI), or $10 \mu \mathrm{M}$ KT5720 (PKAI), or control medium with DMSO.

The kinetics of HSD after two pairings of presynaptic tetanus plus 5 -HT reversed to control levels $(n=7)$ after the acute incubation of chelerythrine $(n=8)$ but remained attenuated after the incubation of DMSO alone $(n=6)$ or KT5720 $(n=6$; Fig. 3$)$. The kinetics of HSD in controls was not affected by the presence of chelerythrine $(n=6)$. The initial synaptic strengths at all sensorimotor synapses (control or paired stimuli), however, were unaffected by the acute $(30 \mathrm{~min}$ ) exposure to chelerythrine or KT5720 (data not shown). Thus, the change in the kinetics of HSD at synapses expressing PA-LTF depends on the activation of PKC, whereas blocking PKC activity does not alter the kinetics of HSD at control synapses.

\section{Constitutive PKC activity is required for maintaining PA-LTF}

The additional stimuli that produce PNA-LTF (the second day of 5-HT applications) activate $\mathrm{PKC}$, which is required for the expression of persistent nonassociative facilitation (Hu et al., 2011). A form of PNA-LTF reverses rapidly to control levels when chelerythrine is applied $24 \mathrm{~h}$ after stimulation (Cai et al., 2011). In addition, activation of PKC may play a role in maintaining LTP or long-term memories in mammals (Shema et al., 2007; Sacktor, 2011; Volk et al., 2013; Jalil et al., 2015). We therefore tested whether constitutive PKC activity was required for the maintenance of PA-LTF (Fig. 4). After recording the EPSP amplitudes on day $7,48 \mathrm{~h}$ after the second pairing of presynaptic tetanus plus 

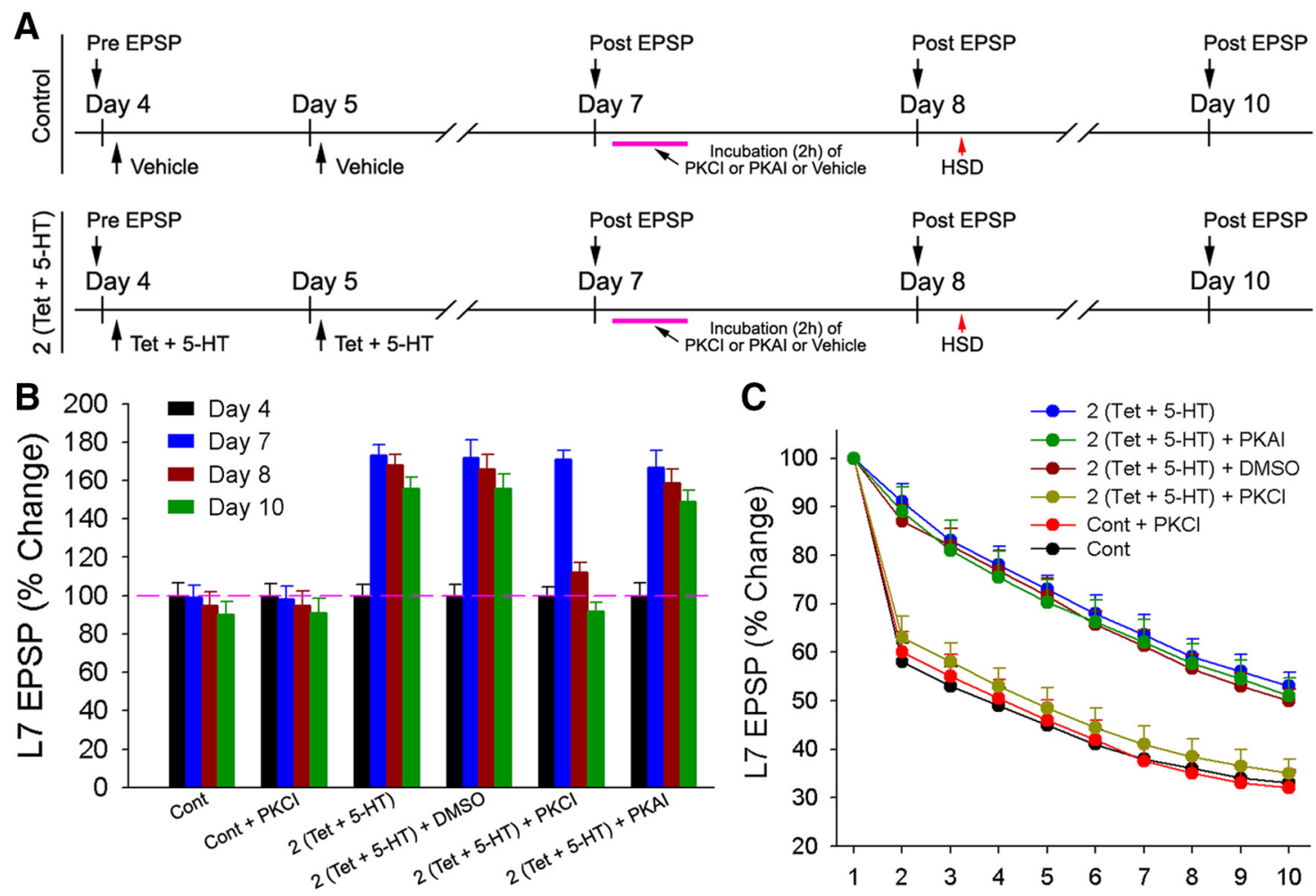

Figure 4. Inhibition of constitutive PKC activity rapidly reverses PA-LTF and the attenuated kinetics of HSD. $\boldsymbol{A}$, Experimental protocol. After monitoring the initial EPSP amplitudes on day 4, cultures were divided into two groups: control and experimental groups [2 (Tet +5 -HT)]. On day 7, the control group was divided into two groups [Cont and Cont + PKCI (2 h application of chelerythrine after testing the EPSP on day 7)], and the experimental group was divided into four groups [2 (Tet + 5-HT), 2 (Tet + 5-HT) + PKCl, or PKAl, or DMSO (2 h applications)]. After testing the EPSP amplitudes on day 8 , sensory neurons were stimulated to produce HSD. The EPSP amplitudes were re-examined on day 10 . $\boldsymbol{B}$, Summary of the changes in the EPSP amplitudes for each treatment indicated that the PKC inhibitor but not the PKA inhibitor reversed PA-LTF. A two-factor ANOVA (treatment and time) indicated a significant effect $\left(F_{(15,138)}=178.547 ; p<0.001\right)$. Individual comparisons indicated that treatment with PKC inhibitor significantly reduced the increase in the EPSP amplitudes by 2 (Tet +5 -HT) on days 8 and 10 compared with the increases evoked by $2($ Tet $+5-\mathrm{HT})$ alone $(F=9.509$ and 13.636 ; all $p$ values $<0.001), 2($ Tet +5 -HT $)+$ PKAl $(F=5.503$ and 8.939 ; all $p$ values $<0.001)$, and $2($ Tet +5 -HT $)+$ DMSO $(F=7.221$ and 11.057 ; all $p$ values $<0.001$ ). The changes in the EPSP amplitudes produced by 2 (Tet $+5-\mathrm{HT})+\mathrm{PKCl}$ were no longer significantly different from those in Cont $(F=0.831$ and 0.14 ; all $p$ values $>0.9$ ) or Cont + PKCI $(F=0.661$ and 0.004 ; all $p$ values $>0.9)$. PA-LTFs evoked by all other 2 (Tet $+5-H T)$ treatments were not significantly different from each other but were significantly different from Cont ( $F$ values between 8.558 and 14.051 ; all $p$ values $<0.001$ ) or Cont + PKCI ( $F$ values between 7.362 and 11.997 ; all $p$ values $<0.001)$. Control baseline was unaffected by treatment with PKC inhibitor. The change in the EPSP amplitude on days 8 and 10 for Cont was not significantly different from those for Cont + PKCI (Fvalues between 0.001 and 0.004 ; all $p$ values $>0.9$ ). C, The kinetics of HSD on day 8 reversed to control levels with 2 (Tet $+5-\mathrm{HT}$ ) + PKCI treatment. HSD kinetics for all other 2 (Tet +5 -HT) treatments remained attenuated compared with controls. A two-factor ANOVA indicated a significant effect on the kinetics of HSD $\left(F_{(40,264)}=3.281 ; p<0.001\right)$. Individual comparisons indicated that HSD kinetics for 2 (Tet +5 -HT) + PKCI was significantly different from those observed for 2 (Tet +5 -HT) $(F=3.32 ; p<0.003)$, 2 (Tet $+5-\mathrm{HT})+\operatorname{PKAI}(F=2.75 ; p<0.01)$, and 2 (Tet $+5-\mathrm{HT})+$ DMSO $(F=4.375 ; p<0.001)$ but not significantly different from that in Cont $(F=0.438 ; p>0.8)$ or Cont $+\operatorname{PKCl}(F=0.047 ; p>0.9)$.

5-HT, cultures (controls or two pairings) were incubated for $2 \mathrm{~h}$ with chelerythrine, KT5720, DMSO alone, or medium alone. The synaptic strengths were re-examined on days 8 and 10 . The kinetics of HSD was also examined on day 8.

The maintenance of PA-LTF required constitutive PKC activity (Fig. 4). Whereas two pairings of presynaptic tetanus plus 5 -HT $(n=10)$ produced a significant increase in the synaptic strength on days 8 and $10(168 \pm 5.6 \%$ and $156 \pm 5.7 \%)$ that was not affected by incubation with DMSO alone $(n=7 ; 166 \pm 7.8 \%$ and $156 \pm 7.4 \% ; p>0.9$ ), incubation with chelerythrine (PKCI) significantly reduced the synaptic strength back to the control baseline $(n=12 ; 112 \pm 5.1 \%$ and $92 \pm 4.4 \% ; p<0.01)$. The synaptic baseline in controls, however, was not affected by incubation with chlerythrine $[95 \pm 6.9 \%$ and $90 \pm 6.8 \%$ for control $(n=9)$ vs $95 \pm 7.3 \%$ and $91 \pm 7.5 \%$ for control plus PKCI $(n=$ 7)]. PA-LTF was not affected by incubation with KT5720 $(n=7$; $159 \pm 6.8 \%$ on day 8 and $149 \pm 5.9 \%$ on day 10 ). Control cultures treated with KT5720 were also not affected and were not significantly different from the other controls (data not shown). The decline in the EPSP amplitudes at synapses expressing PALTF after incubation with chelerythrine resulted in the synaptic strengths that were no longer significantly different from those in controls treated with or without chelerythrine (all $p$ values $>0.9$ ). Thus, the maintenance of the persistent change in the synaptic strength evoked by external stimuli, but not the maintenance of the synaptic baseline, requires constitutive PKC activity.

The attenuated kinetics of HSD accompanying PA-LTF also returned to control levels on day 8 when PA-TLF was reversed by chelerythrine (Fig. 4C), which was not significantly different from the kinetics of HSD observed in control or control plus chelerythrine (all $p$ values $>0.8$ ). Compared with controls, the kinetics of HSD remained attenuated at synapses expressing PALTF when incubated with either KT5720 or DMS0 alone (all $p$ values $<0.003)$. Thus, both the persistent expression of attenuated HSD kinetics and the persistent facilitation produced by paired stimuli require PKC activities.

Homosynaptic activation followed by rapamycin rapidly reverses PA-LTF and the attenuated kinetics of HSD

PNA-LTF expressed at sensorimotor synapses that is evoked by repeated applications of 5-HT is rapidly reversed to the synaptic baseline when heterosynaptic reactivation (5-HT or FMRFa) is 
followed by the protein synthesis inhibitor rapamycin. In contrast, PNA-LTF is not reversed by homosynaptic activation (PTP or HSD) plus rapamycin or anisomycin ( $\mathrm{Hu}$ and Schacher, 2014). We therefore examined whether the homosynaptic or heterosynaptic forms of short-term plasticity immediately followed by the incubation with rapamycin on day 7 reversed PA-LTF and the attenuated kinetics of HSD (Figs. 5A, 6A).

Short-term homosynaptic activation of PTP alone or rapamycin alone on day 7 did not interfere with PA-LTF produced by two pairings of presynaptic tetanus plus $5-\mathrm{HT}$, but incubation with rapamycin immediately after the stimuli inducing PTP on day 7 resulted in the rapid reversal of PA-LTF (Fig. 5B1). The synaptic strength was significantly increased on days 8 and 10 by two pairings $(167 \pm 14.1 \%$ and $155 \pm 13.6 \% ; n=10)$ and remained elevated when two pairings were followed by PTP alone $(n=8 ; 166 \pm 9.6 \%$ and $153 \pm 9.4 \% ; p>0.9)$ or by rapamycin alone $(n=8 ; 164 \pm 15.0 \%$ and $154 \pm 15.0 \% ; p>0.9)$. However, when rapamycin was applied immediately after PTP on day 7 , the increase in the synaptic strength produced by two pairings rapidly reversed to control levels on day 8 and remained so on day 10 $(n=10 ; 104 \pm 8.2 \%$ and $91 \pm 7.6 \%)$ and was no longer significantly different from those of various control groups: PTP alone on day $7(n=8 ; 94 \pm 7.6 \%$ and $91 \pm 6.9 \% ; p>0.9)$ or PTP plus rapamycin on day $7(n=7 ; 93 \pm 7.9 \%$ and $90 \pm 7.7 \% ; p>0.9)$. PTP followed by rapamycin did not significantly affect the synaptic baseline in controls $(p>0.9)$.

Similarly, short-term HSD alone on day 7 did not interfere with PA-LTF produced by two pairings of presynaptic tetanus plus 5 -HT, but incubation with rapamycin immediately after HSD on day 7 resulted in the rapid reversal of PA-LTF (Fig. 5C1). The increase in the synaptic strength was maintained on days 8 and 10 after two pairings ( $n=7 ; 165 \pm 13.1 \%$ and $155 \pm 13.3 \%)$ and was maintained as well when two pairings were followed by HSD alone $(n=8 ; 163 \pm 15.6 \%$ and $154 \pm 15.3 \% ; p>0.9)$. Despite the reduction in the synaptic strength produced by HSD, a decline in the EPSP amplitude back to the original baseline, PA-LTF was unaffected. However, the increases in the synaptic strength produced by two pairings of presynaptic tetanus plus 5-HT rapidly reversed to control levels on day 8 and remained so on day 10 when HSD was immediately followed by incubation with rapamycin on day $7(n=12 ; 116 \pm 9.1 \%$ and $98 \pm 7.4 \%)$, which were no longer significantly different from those of various control groups: HSD alone on day $7(n=7 ; 92 \pm 6.8 \%$ and $91 \pm$ $7.1 \% ; p>0.9)$ or HSD followed by rapamycin on day $7(n=7$; $93 \pm 7.7 \%$ and $90 \pm 8.3 \% ; p>0.9)$. HSD followed by rapamycin did not significantly affect the control synaptic baseline $(p>0.9)$. The addition of a protein synthesis inhibitor immediately after reactivation rapidly reversed the persistent facilitation underlying a form of long-term classical conditioning but had no effect on the control synaptic baseline. Unlike the rapid reversal induced by chelerythrine alone, blockade of protein synthesis by rapamycin alone failed to reverse PA-LTF. Thus, unlike the situation with PNA-LTF, sensorimotor synapses expressing PA-LTF express reconsolidation blockade with homosynaptic reactivation plus rapamycin.

We also examined whether the altered kinetics of HSD accompanied the reversal of PA-LTF when homosynaptic reactivation was followed immediately by rapamycin on day 7 (Fig. 5B2,C2). The kinetics of HSD reversed as the increase in the synaptic strength produced by two pairings of presynaptic tetanus plus 5-HT were reversed after homosynaptic reactivation (PTP or HSD) on day 7 was followed by rapamycin. Reactivation followed by rapamycin reversed HSD kinetics on day 8 such that they were no longer significantly different from the HSD kinetics for controls: rapamycin alone, PTP alone, and HSD alone (all $p$ values $>0.9$ ). Conditions that failed to reverse PA-LTF produced by two pairings of presynaptic tetanus plus 5-HT also failed to reverse the attenuated kinetics of HSD: two pairings plus PTP, HSD, or rapamycin on day 7 ( $p>0.9)$. Thus, both the persistent facilitation and the attenuated kinetics of HSD are affected by interfering with constitutive PKC activity or when the homosynaptic reactivation is followed by a transient inhibition of protein synthesis by rapamycin.

\section{Heterosynaptic activation followed by rapamycin fails to reverse PA-LTF}

We next examined whether heterosynaptic activation (5-HT or FMRFa) alone affected PA-LTF and whether these reactivations induced reconsolidation blockade or a reversal in the attenuated kinetics of HSD when the reactivations were followed immediately by rapamycin on day 7 (Fig. 6). PNA-LTF becomes labile by either brief applications of 5-HT (which activates PKA, PKC, and ERK1/2 signaling) or FMRFa (which activates arachidonic acid and P38 MAP kinase signaling), which under some circumstances can significantly affect the short- and long-term facilitation produced by 5-HT (Guan et al., 2002).

Short-term heterosynaptic reactivation (5-HT or FMRFa) alone on day 7 did not interfere with PA-LTF, and rapamycin added after the reactivations failed to reverse PA-LTF (Fig. $6 B 1, C 1)$. The synaptic strengths were significantly increased on days 8 and 10 by two pairings of presynaptic tetanus plus 5-HT $[n=8,163 \pm 6.1 \%$ and $154 \pm 5.9 \%$ (Fig. $6 B 1$ ); or $n=7,159 \pm$ $9.4 \%$ and $151 \pm 9.4 \%$ (Fig. 6C1)], and that increase was not affected when two pairings were followed by a brief application of 5 -HT alone on day $7(n=7 ; 163 \pm 10.0 \%$ and $151 \pm 9.3 \% ; p>$ $0.8)$ or FMRFa alone on day $7(n=7 ; 161 \pm 12.4 \%$ and $154 \pm$ $12.9 \% ; p>0.8)$. Despite the significant short-term depression in the synaptic strength produced by the application of FMRFa, PA-LTF was unaffected.

The addition of rapamycin after heterosynaptic reactivation (5-HT or FMRFa) on day 7 failed to reverse PA-LTF. The increases in the synaptic strength on days 8 and 10 evoked by two pairings of presynaptic tetanus plus 5-HT were not significantly affected by a brief application of either 5-HT followed by rapamycin $(n=8 ; 155 \pm 10.7 \%$ and $143 \pm 9.8 \% ; p>0.8)$ or by FMRFa followed by rapamycin $(n=11 ; 156 \pm 5.4 \%$ and $147 \pm$ $6.8 \% ; p>0.8)$. The synaptic strength on days 8 and 10 after the heterosynaptic reactivation plus rapamycin on day 7 remained significantly higher than those for various controls: 5-HT alone $(n=7 ; 92 \pm 5.3 \%$ and $90 \pm 4.5 \%$; $p<0.01), 5$-HT followed by rapamycin $(n=6 ; 93 \pm 7.5 \%$ and $91 \pm 7.3 \% ; p<0.01), \mathrm{FMRFa}$ alone $(n=7 ; 92 \pm 7.7 \%$ and $89 \pm 7.5 \% ; p<0.01)$, or $\mathrm{FMRFa}$ followed by rapamycin $(n=6 ; 93 \pm 9.8 \%$ and $90 \pm 8.9 \%$; $p<$ 0.01 ). Thus, heterosynaptic reactivations plus rapamycin fail to induce reconsolidation blockade at sensorimotor synapses expressing a cellular analog of classical conditioning (PA-LTF) but do induce reconsolidation blockade at sensorimotor synapses expressing a cellular analog of nonassociative sensitization (PNALTF; Hu and Schacher, 2014).

We also examined whether heterosynaptic reactivation followed immediately by rapamycin affected the attenuated kinetics of HSD accompanying PA-LTF (Fig. 6C2). HSD kinetics at sensorimotor synapses expressing PA-LTF remained attenuated on day 8 after heterosynaptic reactivation (5-HT or FMRFa), followed by rapamycin on day 7, and was not significantly different from the kinetics of HSD at synapses expressing PA-LTF alone. All cultures treated with 

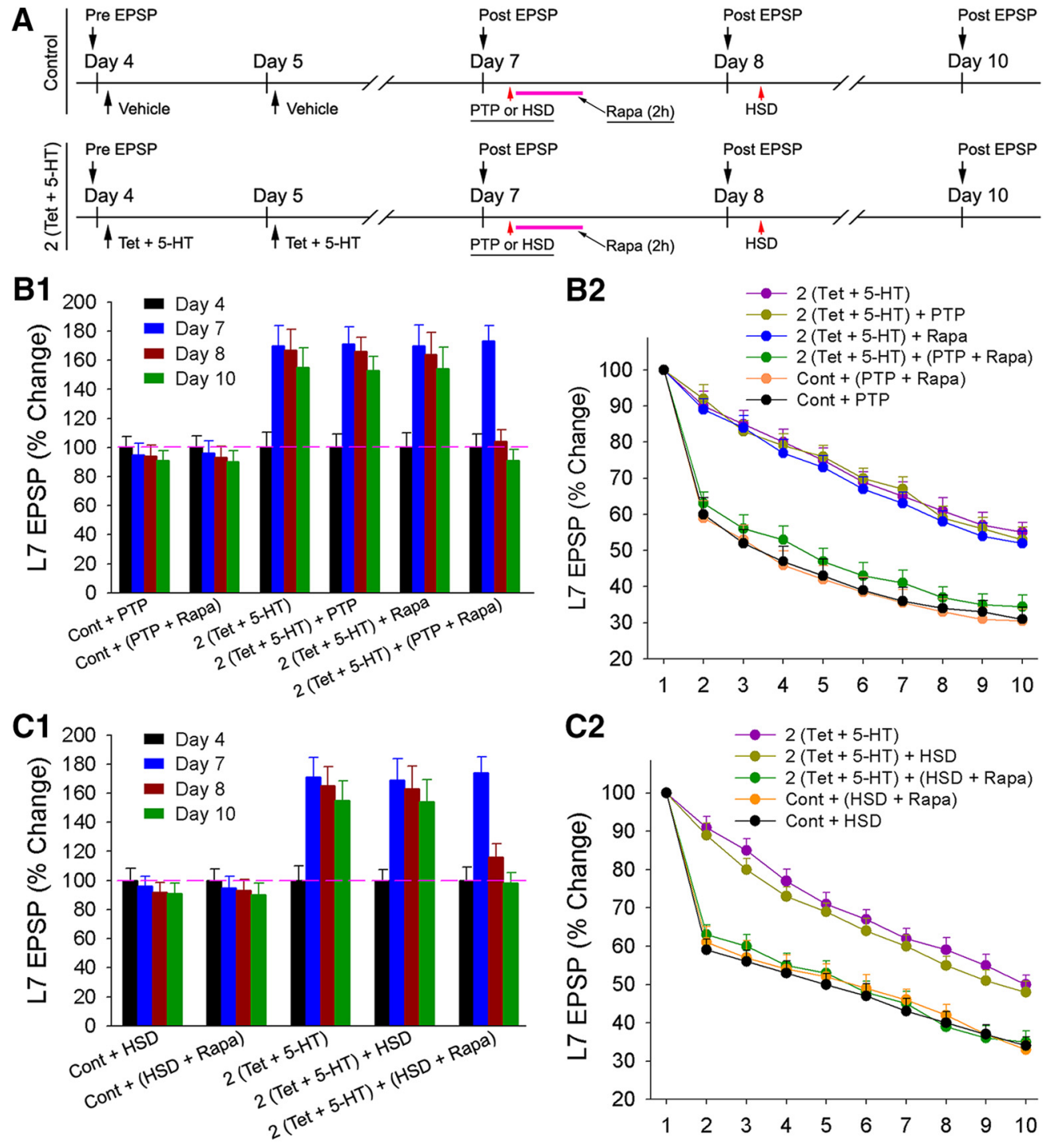

Figure 5. Short-term homosynaptic reactivation fails to interfere with PA-LTF but induces a labile state; reactivation followed by rapamycin rapidly reverses both PA-LTF and the attenuated kinetics of HSD. A, Experimental protocol. After monitoring the EPSP amplitudes on day 7, control cultures were given homosynaptic stimuli (Cont + PTP or Cont + HSD) or rapamycin immediately after the homosynaptic stimuli [Cont + (PTP + Rapa) or Cont + (HSD + Rapa)]. Experimental cultures consisted of 2 (Tet + 5-HT), 2 (Tet + 5-HT) + homosynaptic stimuli, 2 (Tet + 5-HT) + rapamycin, or 2 (Tet +5 -HT) + (homosynaptic stimuli + rapamycin). The EPSP amplitudes were re-examined on days 8 and 10. The kinetics of HSD was determined on day $8 . \mathbf{B 1}$, Summary of the changes in the EPSP amplitudes for each treatment indicated that PA-LTF produced by 2 (Tet +5 -HT) reversed when reactivation of PTP was followed by rapamycin. PTP with or without rapamycin did not affect control baselines. A two-factor ANOVA (treatment and time) indicated a significant difference between the groups $\left(F_{(15,135)}=61.155 ; p<0.001\right)$. Individual comparisons indicated treatment with rapamycin immediately after PTP [2 (Tet + 5-HT) + (PTP + Rapa)] significantly reduced the increases in the EPSP amplitude on days 8 and 10 compared with that evoked by 2 (Tet +5 -HT) alone $(F=3.797$ and 4.15 ; all $p$ values $<0.01), 2($ Tet $+5-H T)+P T P(F=3.21$ and $3.454 ; p<0.05$ and 0.01$)$, and 2 (Tet $+5-H T)+\operatorname{Rapa}(F=3.055$ and $3.552 ; p<0.05$ and 0.01$)$. The change in the EPSP amplitude in 2 (Tet +5 -HT) + (PTP + Rapa) on day 8 or day 10 was no longer significantly different from those in Cont + PTP $(F=0.089$ and $0.002 ;$ all $p$ values $>0.9)$ or Cont $+($ PTP + Rapa) $(F=0.088$ and 0.001 ; all $p$ values $>0.9)$. Conditions that evoked PA-LTF were not significantly different from each other but were significantly greater than the changes in the EPSP amplitudes in Cont + PTP ( F values between 3.243 and 4.56; $p<0.01$ or 0.05 ) or Cont + (PTP + Rapa) (Fvalues between 3.001 and 4.264; $p<0.01$ or 0.05$)$. B2, The kinetics of HSD on day 8 reversed to control levels with 2 (Tet +5 -HT) + (PTP + Rapa). HSD kinetics remained attenuated compared with controls for all other 2 (Tet +5 -HT) treatments. A two-factor ANOVA indicated a significant effect on the kinetics of HSD $\left(F_{(40,360)}=3.614 ; p<0.001\right)$. Individual comparisons indicated that HSD kinetics after 2 (Tet +5 -HT) $+($ PTP + Rapa) were significantly different from those observed in 2 (Tet $+5-\mathrm{HT})(F=3.39 ; p<0.002), 2$ (Tet +5 -HT) + PTP $(F=8.406 ; p<0.001)$, and 2 (Tet +5 -HT) + Rapa $(F=7.577 ; p<0.001)$ but were not significantly different from those in Cont + PTP $(F=1.093 ; p>0.3)$ and Cont + (PTP + Rapa) $(F=0.794 ; p>0.6)$. C1, Summary of the changes in the EPSP amplitudes for each treatment indicated that PA-LTF evoked by 2 (Tet +5 -HT) was reversed by treatment with rapamycin immediately after HSD. HSD with or without rapamycin did not affect control baselines. A two-factor ANOVA (treatment and time) indicated a significant difference between the groups $\left(F_{(12,108)}=35.995 ; p<0.001\right)$. Treatment with rapamycin immediately after HSD $[2$ (Tet +5 -HT) $+($ HSD + Rapa) $]$ significantly reduced the increases in the EPSP amplitudes on days 8 and 10 compared with those produced by 2 (Tet +5 -HT) alone $(F=2.634$ and $4.024 ; p<0.05$ and 0.01$)$ and 2 (Tet +5 -HT) + HSD $(F=$ 2.592 and 4.211; $p<0.05$ and 0.01 ). The EPSP amplitudes on days 8 and 10 in 2 (Tet $+5-H T)+(H S D+$ Rapa) were no longer significantly different from those in Cont + HSD $(F=0.615$ and 0.063 ; all $p$ values $>0.6$ ) or Cont + (HSD + Rapa) $(F=0.571$ and 0.058 ; all $p$ values $>0.7)$. PA-LTF evoked by 2 (Tet +5 -HT) was not significantly different from that evoked by 2 (Tet +5 -HT) + HSD, but the changes in the EPSP amplitudes were significantly higher than those in Cont + HSD ( $F$ values between 4.034 and 4.588; all $p$ values $<0.01)$ or Cont + (HSD + Rapa) $(F$ values between 3.998 and 4.481; all $p$ values $<0.01$ ). (2) The kinetics of HSD on day 8 reversed to control levels with 2 (Tet $+5-H T)+(H S D+$ Rapa). HSD kinetics remained attenuated compared with controls for all other 2 (Tet +5 -HT) treatments. A two-factor ANOVA indicated a significant effect on the kinetics of HSD $\left(F_{(32,288)}=8.956 ; p<0.001\right)$. Individual comparisons indicated that HSD kinetics for 2 (Tet +5 -HT) + (HSD + Rapa) were significantly different from those observed for 2 (Tet $+5-H T)(F=14.289 ; p<0.001)$ and $2($ Tet $+5-H T)+H S D(F=11.552 ; p<0.001)$ but not significantly different from those in Cont + HSD $(F=1.056 ; p>0.1)$ or Cont + (HSD + Rapa) $(F=1.012 ; p>0.1)$. Treatments that did not attenuate HSD kinetics were not significantly different from each other ( $F$ values between 0.063 and 0.101 ; all $p$ values $>0.9$ ). 

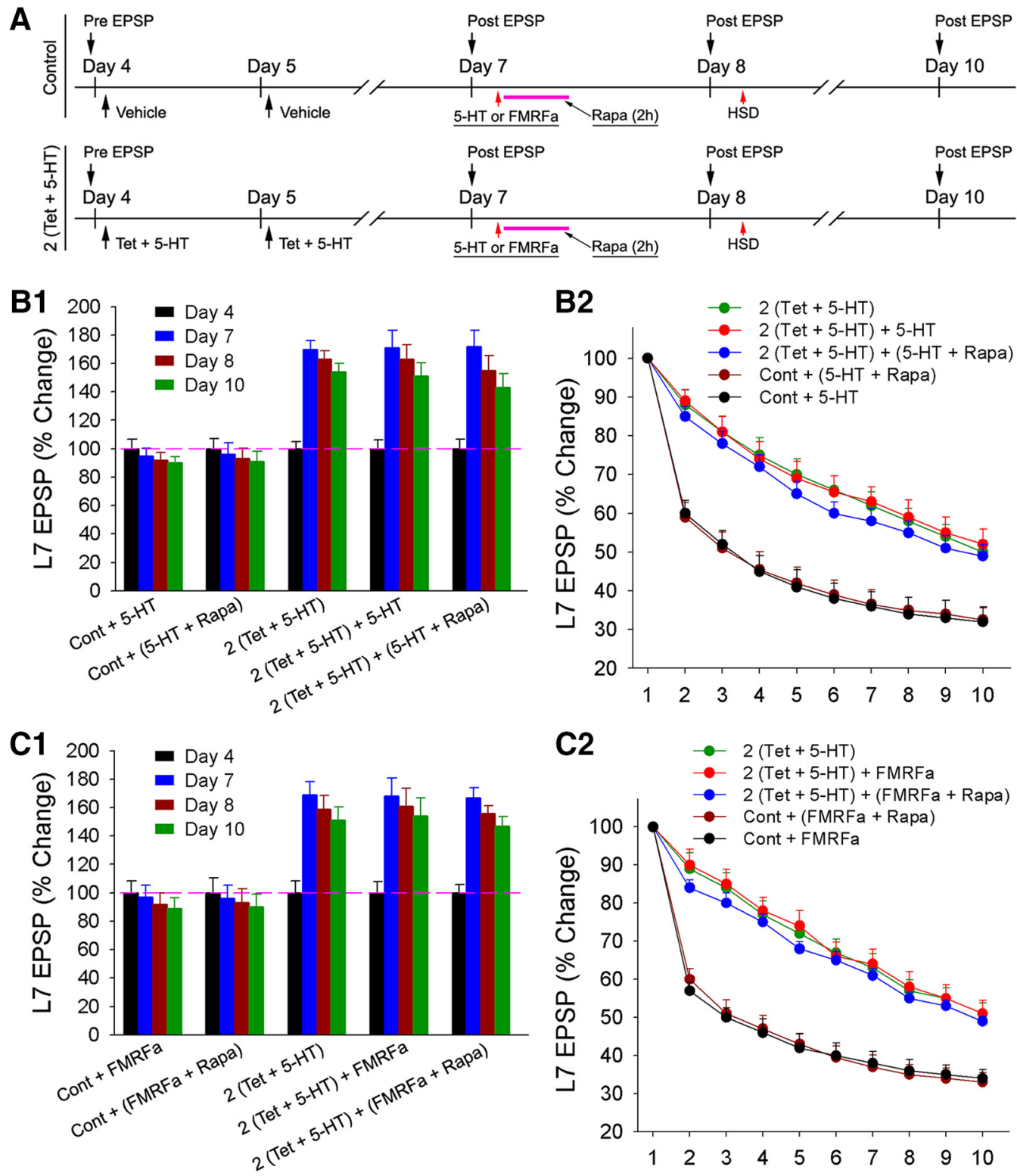

Figure 6. Short-term heterosynaptic reactivation fails to interfere with PA-LTF or to induce a labile state; PA-LTF and the attenuated kinetics of HSD do not reverse after reactivation followed by rapamycin. $A$, Experimental protocol. After monitoring the EPSP amplitudes on day 7, control cultures were divided into four groups: Cont $+5-\mathrm{HT}$, Cont $+\mathrm{FMRFa}$, Cont + (5-HT + Rapa), and Cont + (FMRFa + Rapa). Experimental cultures were divided into five groups: 2 (Tet + 5-HT), 2 (Tet + 5-HT) + 5-HT or FMRFa, 2 (Tet + 5-HT) + (5-HT or FMRFa + Rapa). The EPSP amplitudes were re-examined on days 8 and 10. The kinetics of HSD was determined on day 8 . B1, Summary of the changes in the EPSP amplitudes indicated that treatment with 5-HT alone or 5-HT plus rapamycin on day 7 did not significantly affect PA-LTF evoked by 2 (Tet $+5-\mathrm{HT}$ ). A brief application of 5-HT with or without rapamycin did not affect control baselines. A two-factor ANOVA (treatment and time) indicated a significant difference between the groups $\left(F_{(12,93)}=23.656 ; p<0.001\right)$. Individual comparisons indicated that PA-LTF produced by all 2 (Tet +5 -HT) treatments were not significantly different from each other on days 7, 8, and 10 but were significantly different from those in Cont +5 -HT ( $F$ values between 6.097 and 9.442 ; all $p$ values $<0.01$ ) or Cont $+(5-H T+R a p a)$ ( $F$ values between 5.495 and 8.545 ; all $p$ values $<0.01)$. B2, The attenuated kinetics of HSD on day 8 evoked by all 2 (Tet $+5-\mathrm{HT}$ ) treatments remained attenuated compared with all controls. A two-factor ANOVA indicated a significant effect on the kinetics of HSD $\left(F_{(32,248)}=5.939 ; p<\right.$ 0.001). Individual comparisons indicated that HSD kinetics for all 2 (Tet $+5-\mathrm{HT}$ ) groups were not significantly different from each other but were significantly different from those observed in Cont +5 -HT ( $F$ values between 4.779 and 9.684 ; all $p$ values $<0.001)$ or Cont $+(5-H T+R a p a)$ ( $F$ values between 6.987 and 13.647 ; all $p$ values $<0.001)$. C1, Summary of the changes in EPSP amplitudes for each group indicated that treatment with rapamycin immediately after FMRFa did not significantly affect PA-LTF evoked by 2 (Tet +5 -HT). FMRFa with or without rapamycin did not affect control baselines. A two-factor ANOVA (treatment and time) indicated a significant difference between the groups $\left(F_{(12,99)}=67.491 ; p<\right.$ 0.001). Individual comparisons indicated that PA-LTF produced by all 2 (Tet +5 -HT) treatments were not significantly different from each other on day 7 , day 8 and day 10 , but were significantly different from those in Cont + FMRFa ( $F$ values between 5.291 and 7.81 ; all $p$ values $<0.01$ ) or Cont $+(F M R F a+R a p a)$ ( $F$ values between 4.771 and 7.582 ; all $p$ values $<0.01$ ). C2, The kinetics of HSD on day 8 evoked by all 2 (Tet $+5-H T$ ) treatments were attenuated compared with all controls. A two-factor ANOVA indicated a significant effect on the kinetics of HSD $\left(F_{(32,264)}=8.18 ; p<0.001\right)$. Individual comparisons indicated that HSD kinetics for all 2 (Tet +5 -HT) groups were not significantly different from each other but were significantly different from those observed in Cont + FMRFa ( $F$ values between 16.433 and 20.517 ; all $p$ values $<0.001)$ or Cont $+(F M R F a+$ Rapa) $(F$ values between 9.134 and 11.404 ; all $p$ values $<0.001$ ). 
two pairings of stimuli expressed the attenuated kinetics of HSD compared with the various controls. Thus, the heterosynaptic reactivation alone or pairing with rapamycin that fails to reverse PA-LTF also fails to reverse the attenuated kinetics of HSD.

\section{Discussion}

The sensorimotor synapse of Aplysia expresses at least two forms of persistent facilitation: (1) a nonassociative form (PNA-LTF; Hu et al., 2011) evoked by 10 spaced applications of 5-HT given over $2 \mathrm{~d}$ that is a cellular analog of long-term sensitization (Frost et al., 1985) and (2) an associative form (PA-LTF) produced by two pairings of presynaptic tetanus plus 5-HT given over $2 \mathrm{~d}$ that is a cellular analog of classical conditioning (Buonomano and Byrne, 1990).

Both forms share common properties: each expresses a "transient" long-term form (requiring new macromolecular synthesis; Montarolo et al., 1986; Hu et al., 2007) produced by $1 \mathrm{~d}$ of stimulation and a more persistent form evoked by $2 \mathrm{~d}$ of stimuli. Both forms can express short-term plasticity along with persistent plasticity, indicating that the persistent change in the synaptic strength represents a new baseline that can be modulated with additional stimuli. Both forms require constitutive PKC activity for maintenance, since the transient blockade of PKC activation results in their rapid reversal (Cai et al., 2011; Chen et al., 2014). PKC activation is required for the initiation and consolidation of long-term plasticity and memory in mammals, but the roles of various $\mathrm{PKC}$ isoforms (especially $\mathrm{PKM} \zeta$ ) in maintaining persistent plasticity or in reconsolidation remain controversial (Shema et al., 2007; Sacktor, 2011; Volk et al. 2013; Jalil et al., 2015). In addition, the increased synaptic strength returns to the original baseline when the persistent plasticity reverses. The original baseline is unaffected when reactivation is paired with the same inhibitors that reverse the persistent facilitation (Hu and Schacher, 2014). The labile cellular/molecular processes that maintain the synaptic plasticity required for long-term memory appear to be independent of the mechanisms required for maintaining the original synaptic baseline.

The two forms of persistent facilitation have important differences. Sensorimotor synapses expressing PA-LTF also express an attenuated form of HSD. In contrast, sensorimotor synapses expressing PNA-LTF have HSD kinetics indistinguishable from naive synapses (Hu and Schacher, 2014). Another important difference is the nature of the reactivations that evoke reconsolidation blockade when specific reactivations are paired with the protein synthesis inhibitor rapamycin. Heterosynaptic reactivation (5-HT or FMRFa) plus rapamycin produced the rapid reversal of PNALTF (Hu and Schacher, 2014). In contrast, homosynaptic reactivation (HSD or PTP) plus rapamycin evoked the rapid reversal of PA-LTF. Thus, two forms of persistent facilitation expressed at the same synapse, where each form contributes to different forms of learning and memory, become labile with different reactivations. These results extend behavioral studies in mammalian systems where reactivations plus rapamycin lead to a rapid reversal of the long-term memory or plasticity (Stoica et al., 2011; Li et al., 2013; Huynh et al., 2014, Lopez et al., 2015).

\section{PKC activity affects the kinetics of HSD and PA-LTF}

The expression of PA-LTF was accompanied by a significant and persistent attenuation in the kinetics of HSD. This attenuation was not observed when synapses express PNA-LTF (Hu and Schacher, 2014). After an acute incubation with a PKC inhibitor, synapses continued to express PA-LTF but showed HSD kinetics comparable to controls. Since HSD is caused primarily by a decline in evoked transmitter release from the presynaptic terminals (Eliot et al., 1994; Armitage and Siegelbaum, 1998; Royer et al., 2000; Gover et al., 2002; Zhao and Klein, 2002; Wan et al., 2012; Malkinson and Spira, 2013), our results suggest that each action potential in the sensory neuron increases PKC activity, preserving a relatively high rate of release. Such increases in PKC activity led to attenuated HSD kinetics when naive sensorimotor synapses were either stimulated to fire single action potentials in the presence of 5-HT or electrically stimulated to fire two to four action potentials at $20 \mathrm{~Hz}$ at regular intervals (Sun and Schacher, 1996b; Wan et al., 2012). One long-term consequence of the stimuli that produce PA-LTF might be to change the handling of calcium-dependent activation of PKC such that evoked transmitter release at sensorimotor synapses with each action potential simulates the presence of 5-HT. Intracellular free calcium levels might increase via changes in the number or distribution of voltage-gated channels or in the level of calcium released from intracellular stores (Billups and Forsythe, 2002; Dunn and Syed, 2006; Ahmed and Siegelbaum, 2009). Calcium-dependent PKC near synaptic calcium channels might increase as a result of changes in the expression or post-translational modification of adaptor proteins such as PICK1 (Wan et al., 2012; Dargaei et al., 2015), resulting in more efficient coupling of PKC activation with each action potential and neurotransmitter release (Malkinson and Spira, 2013).

Constitutive PKC activity maintains both PNA-LTF (Cai et al., 2011; Bougie et al., 2012) and PA-LTF. Thus, besides the changes in PKC activation at the sensory neuron terminals with each action potential, changes evoked by constitutive PKC activity in other compartments of the sensory neuron and/or L7 contribute to the maintenance of PA-LTF. Constitutive PKC activity is also required for maintaining long-term sensitization in Aplysia, which can then be reinstated with additional sensitizing stimuli (Chen et al., 2014). In contrast, PKA activity, critical for initiating both nonassociative and associative forms of plasticity at sensorimotor synapses (Kandel et al., 1983; Walters and Byrne, 1983), does not appear to be critical for maintaining PA-LTF.

Constitutive PKC activity also maintains the attenuated kinetics of HSD. Activity of one or more of the three PKC isoforms in Aplysia (Sossin, 2007; Farah and Sossin, 2011) may regulate distinct processes in all cellular compartments of the sensory neuron and L7 (Antonov et al., 2003; Hu et al., 2010; Bougie et al., 2012; Cai et al., 2012; Fiumara et al., 2015) required to maintain both PA-LTF and PNA-LTF, and the altered kinetics of HSD that accompany PA-LTF. Future studies using cell-specific manipulations of the different PKC isoforms will allow us to explore the cellular localization and role of each PKC isoform in regulating PA-LTF and the altered kinetics of HSD and PNA-LTF.

\section{Homosynaptic reactivation induces a labile state at synapses expressing PA-LTF}

5-HT is a common stimulus in the induction of both PNALTF and PA-LTF, but reactivation by 5 -HT induced a labile state at sensorimotor synapses expressing PNA-LTF but not at synapses expressing PA-LTF. Homosynaptic reactivation induced a labile state only at sensorimotor synapses expressing PA-LTF but not at synapses expressing PNA-LTF (Hu and Schacher, 2014).

One explanation for this dichotomy is that each reactivation on day 7 leads to post-translational modifications of two different sets of molecules: a common set that produces the short-term plasticity and a newly synthesized set of molecules, some of which are uniquely associated with each form of persistent plasticity and provides each form with unique features (a form of metaplasticity; McCamphill et al., 2015). 
Homosynaptic reactivation at synapses expressing PA-LTF, either a weak (HSD) or identical (PTP) versions of the conditioned stimulus, evokes different responses than at naive synapses or synapses expressing PNA-LTF. With low-frequency stimulation, increases in the activation of specific isoforms of PKC at sensorimotor synapses, coupled with the transient blockade of protein synthesis in various compartments in both the sensory neurons and L7, might affect processes required for maintaining both PA-LTF and the attenuated kinetics of HSD.

The other homosynaptic reactivation PTP has pleiotropic actions on both the presynaptic sensory neuron and postsynaptic motor neuron that include the release of calcium from intracellular stores in both presynaptic and postsynaptic compartments, activation of CaMK and MAPK, and calcium-dependent protein degradation (Schaffhausen et al., 2001; Jin and Hawkins, 2003; Khoutorsky and Spira, 2009). These presynaptic and postsynaptic activations at sensorimotor synapses expressing PA-LTF may modify new substrates that are part of the molecular cascade that maintains PA-LTF. The modifications of these new substrates by the homosynaptic reactivations may induce the labile state. When the reactivations are paired with the transient inhibition of the synthesis of critical proteins (both in the cell bodies and the synaptic terminals), these substrate modifications cause a disruption in the processes required for maintaining both the plasticity and the attenuated kinetics of HSD.

In contrast, heterosynaptic activity fails to induce a labile state at synapses expressing PA-LTF. Heterosynaptic activation (5-HT or FMRFa) is acting on the sensory neurons and L7 that have been exposed only twice to 5-HT. The prior stimulus history (two exposures to 5-HT that contribute to PA-LTF) may be inadequate for a heterosynaptic reactivation to induce a labile state. But 10 exposures to 5-HT, which produce PNA-LTF as a result of cell-specific responses in both the sensory neuron (sensorin expression and secretion; Hu et al., 2011) and L7 (expression of specific bZIP transcription factors; $\mathrm{Hu}$ et al., 2015), may adequately alter the cells' responses to subsequent heterosynaptic activity (including FMRFa) to induce a labile state. Future studies will examine the nature of the labile state produced by selective reactivations for both forms of persistent plasticity.

\section{References}

Ahmed MS, Siegelbaum SA (2009) Recruitment of N-type Ca(2+) channels during LTP enhances low release efficacy of hippocampal CAl perforant path synapses. Neuron 63:372-385. CrossRef Medline

Alberini CM, Kandel ER (2014) The regulation of transcription in memory consolidation. Cold Spring Harb Perspect Biol 7:a021741. CrossRef Medline

Antonov I, Antonova I, Kandel ER, Hawkins RD (2003) Activity-dependent presynaptic facilitation and hebbian LTP are both required and interact during classical conditioning in Aplysia. Neuron 37:135-147. CrossRef Medline

Armitage BA, Siegelbaum SA (1998) Presynaptic induction and expression of homosynaptic depression at Aplyia sensorimotor neuron synapses. J Neurosci 18:8770-8779. Medline

Bailey CH, Montarolo P, Chen M, Kandel ER, Schacher S (1992) Inhibitors of protein and RNA synthesis block structural changes that accompany long-term heterosynaptic plasticity in Aplysia. Neuron 9:749-758. CrossRef Medline

Billups B, Forsythe ID (2002) Presynaptic mitochondrial calcium sequestration influences transmission at mammalian central synapses. J Neurosci 22:5840-5847. Medline

Bougie JK, Cai D, Hastings M, Farah CA, Chen S, Fan X, McCamphill PK, Glanzman DL, Sossin WS (2012) Serotonin-induced cleavage of the atypical protein kinase C Apl III in Aplysia. J Neurosci 32:14630-14640. CrossRef Medline

Brunelli M, Castellucci V, Kandel ER (1976) Synaptic facilitation and behavioral sensitization in Aplysia: possible role of serotonin and cyclic AMP. Science 194:1178-1181. CrossRef Medline
Buonomano DV, Byrne JH (1990) Long-term synaptic changes produced by a cellular analog of classical conditioning in Aplysia. Science 249: 420-423. CrossRef Medline

Byrne JH, Hawkins RD (2015) Nonassociative learning in invertebrates. Cold Spring Harb Perspect Biol 7 pii: a021675. CrossRef Medline

Cai D, Pearce K, Chen S, Glanzman DL (2011) Protein kinase M maintains long-term sensitization and long-term facilitation in Aplysia. J Neurosci 31:6421-6431. CrossRef Medline

Cai D, Pearce K, Chen S, Glanzman DL (2012) Reconsolidation of longterm memory in Aplysia. Curr Biol 22:1783-1788. CrossRef Medline

Carew TJ, Walters ET, Kandel ER (1981) Associative learning in Aplysia: cellular correlates supporting a conditioned fear hypothesis. Science 211: 501-504. CrossRef Medline

Carew TJ, Hawkins RD, Kandel ER (1983) Differential classical conditioning of a defensive withdrawal reflex in Aplysia californica. Science 219: 397-400. CrossRef Medline

Chen S, Cai D, Pearce K, Sun PY, Roberts AC, Glanzman DL (2014) Reinstatement of long-term memory following erasure of its behavioral and synaptic expression in Aplysia. eLife 3:e03896. CrossRef Medline

Dargaei Z, Standage D, Groten CJ, Blohm G, Magoski NS (2015) $\mathrm{Ca}^{2+}$. induced uncoupling of Aplysia bag cell neurons. J Neurophysiol 113: 808-821. CrossRef Medline

Da Silva WC, Cardoso G, Bonini JS, Benetti F, Izquierdo I (2013) Memory reconsolidation and its maintenance depend on L-voltage-dependent calcium channels and CaMKII functions regulating protein turnover in the hippocampus. Proc Natl Acad Sci U S A 110:6566-6570. CrossRef Medline

Dong C, Upadhya SC, Ding L, Smith TK, Hegde AN (2008) Proteasome inhibition enhances the induction and impairs the maintenance of longphase long-term potentiation. Learn Mem 15:335-347. CrossRef Medline

Dunn TW, Syed NI (2006) Ryanodine receptor-transmitter release site coupling increases quantal size in a synapse-specific manner. Eur J Neurosci 24:1591-1605. CrossRef Medline

Eliot LS, Kandel ER, Hawkins RD (1994) Modulation of spontaneous transmitter release during depression and posttetanic potentiation of Aplysia sensory-motor neuron synapses isolated in culture. J Neurosci 14: 3280-3292. Medline

Farah CA, Sossin WS (2011) A new mechanism of action of a C2 domainderived novel PKC inhibitor peptide. Neurosci Lett 504:306-310. CrossRef Medline

Fiumara F, Rajasethupathy P, Antonov I, Kosmidis S, Sossin WS, Kandel ER (2015) MicroRNA-22 gates long-term heterosynaptic plasticity in Aplysia through presynaptic regulation of CPEB and downstream targets. Cell Rep 11:1866-1875. CrossRef Medline

Frost WN, Castellucci VF, Hawkins RD, Kandel ER (1985) Monosynaptic connections made by the sensory neurons of the gill- and siphonwithdrawal reflex in Aplysia participate in the storage of long-term memory for sensitization. Proc Natl Acad Sci U S A 82:8266-8269. CrossRef Medline

Glanzman DL, Mackey SL, Hawkins RD, Dyke AM, Lloyd PE, Kandel ER (1989) Depletion of serotonin in the nervous system of Aplysia reduces the behavioral enhancement of gill withdrawal as well as the heterosynaptic facilitation produced by tail shock. J Neurosci 9:4200-4213. Medline

Gover TD, Jiang XY, Abrams TW (2002) Persistent, exocytosis-independent silencing of release sites underlies homosynaptic depression at sensory synapses in Aplysia. J Neurosci 22:1942-1955. Medline

Guan Z, Giustetto M, Lomvardas S, Kim JH, Miniaci MC, Schwartz JH, Thanos D, Kandel ER (2002) Integration of long-term-memory-related synaptic plasticity involves bidirectional regulation of gene expression and chromatin structure. Cell 111:483-493. CrossRef Medline

Hawkins RD, Abrams TW, Carew TJ, Kandel ER (1983) A cellular mechanism of classical conditioning in Aplysia: activity-dependent amplification of presynaptic facilitation. Science 219:400-405. CrossRef Medline

Hu JY, Schacher S (2014) Persistent long-term facilitation at an identified synapse becomes labile with activation of short-term heterosynaptic plasticity. J Neurosci 34:4776-4785. CrossRef Medline

Hu JY, Chen Y, Schacher S (2007) Protein kinase C regulates local synthesis and secretion of a neuropeptide required for activity-dependent longterm synaptic plasticity. J Neurosci 27:8927-8939. CrossRef Medline

Hu JY, Chen Y, Bougie JK, Sossin WS, Schacher S (2010) Aplysia cell adhesion molecule and a novel protein kinase $\mathrm{C}$ activity in the postsynaptic 
neuron are required for presynaptic growth and initial formation of specific synapses. J Neurosci 30:8353-8366. CrossRef Medline

Hu JY, Baussi O, Levine A, Chen Y, Schacher S (2011) Persistent long-term synaptic plasticity requires activation of a new signaling pathway by additional stimuli. J Neurosci 31:8841-8850. CrossRef Medline

Hu JY, Levine A, Sung YJ, Schacher S (2015) cJun and CREB2 in the postsynaptic neuron contribute to persistent long-term facilitation at a behaviorally relevant synapse. J Neurosci 35:386-395. CrossRef Medline

Huynh TN, Santini E, Klann E (2014) Requirement of mammalian target of rapamycin complex 1 downstream effectors in cued fear memory reconsolidation and its persistence. J Neurosci 34:9034-9039. CrossRef Medline

Jalil SJ, Sacktor TC, Shouval HZ (2015) Atypical PKCs in memory maintenance: the roles of feedback and redundancy. Learn Mem 22:344-353. CrossRef Medline

Jin I, Hawkins RD (2003) Presynaptic and postsynaptic mechanisms of a novel form of homosynaptic potentiation at Aplysia sensorimotor synapses. J Neurosci 23:7288-7297. Medline

Johansen JP, Cain CK, Ostroff LE, LeDoux JE (2011) Molecular mechanisms of fear learning and memory. Cell 147:509-524. CrossRef Medline

Kaang BK, Choi JH (2011) Protein degradation during reconsolidation as a mechanism for memory reorganization. Front Behav Neurosci 5:2. CrossRef Medline

Kandel ER (2001) The molecular biology of memory storage: a dialogue between genes and synapses. Science 294:1030-1038. CrossRef Medline

Kandel ER, Abrams T, Bernier L, Carew TJ, Hawkins RD, Schwartz JH (1983) Classical conditioning and sensitization share aspects of the same molecular cascade in Aplysia. Cold Spring Harb Symp Quant Biol $48 \mathrm{Pt}$ 2:821-830.

Kelly A, Laroche S, Davis S (2003) Activation of mitogen-activated protein kinase/extracellular signal-regulated kinase in hippocampal circuitry is required for consolidation and reconsolidation of recognition memory. J Neurosci 23:5354-5360. Medline

Khoutorsky A, Spira ME (2009) Activity-dependent calpain activation plays a critical role in synaptic facilitation and post-tetanic potentiation. Learn Mem 16:129-141. CrossRef Medline

Lee SH, Kwak C, Shim J, Kim JE, Choi SL, Kim HF, Jang DJ, Lee JA, Lee K, Lee CH, Lee YD, Miniaci MC, Bailey CH, Kandel ER, Kaang BK (2012) A cellular model of memory reconsolidation involves reactivation-induced destabilization and restabilization at the sensorimotor synapse in Aplysia. Proc Natl Acad Sci U S A 109:14200-14205. CrossRef Medline

Li Y, Meloni EG, Carlezon WA Jr, Milad MR, Pitman RK, Nader K, Bolshakov VY (2013) Learning and reconsolidation implicate different synaptic mechanisms. Proc Natl Acad Sci U S A 110:4798-4803. CrossRef Medline

Lopez J, Gamache K, Schneider R, Nader K (2015) Memory retrieval requires ongoing protein synthesis and NMDA receptor activity-mediated AMPA receptor trafficking. J Neurosci 35:2465-2475. CrossRef Medline

Lubin FD, Sweatt JD (2007) The IkappaB kinase regulates chromatin structure during reconsolidation of conditioned fear memories. Neuron 55: 942-957. CrossRef Medline

Mackey SL, Kandel ER, Hawkins RD (1989) Identified serotonergic neurons LCB1 and RCB1 in the cerebral ganglia of Aplysia produce presynaptic facilitation of siphon sensory neurons. J Neurosci 9:4227-4235. Medline

Malkinson G, Spira ME (2013) Release properties of individual presynaptic boutons expressed during homosynaptic depression and heterosynaptic facilitation of the Aplysia sensorimotor synapse. Front Cell Neurosci 7:165. Medline

Marinesco S, Wickremasinghe N, Carew TJ (2006) Regulation of behavioral and synaptic plasticity by serotonin release within local modulatory fields in the CNS of Aplysia. J Neurosci 26:12682-12693. CrossRef Medline

McCamphill PK, Farah CA, Anadolu MN, Hoque S, Sossin WS (2015) Bidirectional regulation of eEF2 phosphorylation controls synaptic plasticity by decoding neuronal activity patterns. J Neurosci 35:4403-4417. CrossRef Medline

McGaugh JL (2000) Memory-a century of consolidation. Science 287: 248-251. CrossRef Medline

Montarolo PG, Goelet P, Castellucci VF, Morgan J, Kandel ER, Schacher S (1986) A critical period for macromolecular synthesis in long-term het- erosynaptic facilitation in Aplysia. Science 234:1249-1254. CrossRef Medline

Morris RG, Inglis J, Ainge JA, Olverman HJ, Tulloch J, Dudai Y, Kelly PA (2006) Memory reconsolidation: sensitivity of spatial memory to inhibition of protein synthesis in dorsal hippocampus during encoding and retrieval. Neuron 50:479-489. CrossRef Medline

Nader K, Schafe GE, Le Doux JE (2000) Fear memories require protein synthesis in the amygdala for reconsolidation after retrieval. Nature 406: 722-726. CrossRef Medline

Pinsker HM, Hening WA, Carew TJ, Kandel ER (1973) Long-term sensitization of a defensive withdrawal reflex in Aplysia. Science 182:1039-1042. CrossRef Medline

Royer S, Coulson RL, Klein M (2000) Switching off and on of synaptic sites at Aplysia sensorimotor synapses. J Neurosci 20:626-638. Medline

Sacktor TC (2011) How does PKMzeta maintain long-term memory? Nat Rev Neurosci 12:9-15. CrossRef Medline

Schacher S, Castellucci VF, Kandel ER (1988) cAMP evokes long-term facilitation in Aplysia sensory neurons that requires new protein synthesis. Science 240:1667-1669. CrossRef Medline

Schacher S, Wu F, Sun ZY (1997) Pathway-specific synaptic plasticity: activity-dependent enhancement and suppression of long-term heterosynaptic facilitation at converging inputs on a single target. J Neurosci 17:597-606. Medline

Schaffhausen JH, Fischer TM, Carew TJ (2001) Contribution of postsynaptic $\mathrm{Ca}^{2+}$ to the induction of post-tetanic potentiation in the neural circuit for siphon withdrawal in Aplysia. J Neurosci 21:1739-1749. Medline

Shema R, Sacktor TC, Dudai Y (2007) Rapid erasure of long-term memory associations in the cortex by an inhibitor of PKM . Science 317:951-953. CrossRef Medline

Sossin WS (2007) Isoform specificity of protein kinase Cs in synaptic plasticity. Learn Mem 14:236-246. CrossRef Medline

Squire LR, Alvarez P (1995) Retrograde amnesia and memory consolidation: a neurobiological perspective. Curr Opin Neurobiol 5:169-177. CrossRef Medline

Stoica L, Zhu PJ, Huang W, Zhou H, Kozma SC, Costa-Mattioli M (2011) Selective pharmacogenetic inhibition of mammalian target of Rapamycin complex I (mTORC1) blocks long-term synaptic plasticity and memory storage. Proc Natl Acad Sci U S A 108:3791-3796. CrossRef Medline

Sun ZY, Schacher S (1996a) Tetanic stimulation and cyclic adenosine monophosphate regulate segregation of presynaptic inputs on a common postsynaptic target neuron in vitro. J Neurobiol 29:183-201. CrossRef Medline

Sun ZY, Schacher S (1996b) Development of short-term heterosynaptic facilitation at Aplysia sensorimotor synapses in vitro is accompanied by changes in the functional expression of presynaptic serotonin receptors. J Neurophysiol 76:2250-2261. Medline

Villareal G, Li Q, Cai D, Fink AE, Lim T, Bougie JK, Sossin WS, Glanzman DL (2009) Role of protein kinase $C$ in the induction and maintenance of serotonin-dependent enhancement of the glutamate response in isolated siphon motor neurons of Aplysia californica. J Neurosci 29:5100-5107. CrossRef Medline

Volk LJ, Bachman JL, Johnson R, Yu Y, Huganir RL (2013) PKM-zeta is not required for hippocampal synaptic plasticity, learning and memory. Nature 493:420-423. CrossRef Medline

Walters ET, Byrne JH (1983) Associative conditioning of single sensory neurons suggests a cellular mechanism for learning. Science 219:405-408. CrossRef Medline

Wan Q, Jiang XY, Negroiu AM, Lu SG, McKay KS, Abrams TW (2012) Protein kinase $\mathrm{C}$ acts as a molecular detector of firing patterns to mediate sensory gating in Aplysia. Nat Neurosci 15:1144-1152. CrossRef Medline

Zhang Y, Smolen P, Baxter DA, Byrne JH (2010) The sensitivity of memory consolidation and reconsolidation to inhibitors of protein synthesis and kinases: computational analysis. Learn Mem 17:428-439. CrossRef Medline

Zhao Y, Klein M (2002) Modulation of the readily releasable pool of transmitter and of excitation-secretion coupling by activity and by serotonin at Aplysia sensorimotor synapses in culture. J Neurosci 22:10671-10679. Medline 